Vittalle - Revista de Ciências da Saúde v. 30, n. 1 (2018) 63-71


\title{
Proposta de intervenção ensino-serviço de Práticas Integrativas e Complementares
}

\author{
Ângela Kemel Zanella ${ }^{a^{*}}$; Caroline C. Ramires ${ }^{\mathrm{b}}$; Cristina Peres Rocco ${ }^{\mathrm{c}}$; \\ Morgana D. da Silva, ${ }^{\mathrm{a}, \mathrm{b}, *}$ \\ ${ }^{\text {a }}$ Curso de Fisioterapia, Universidade Federal do Pampa, Uruguaiana, RS, Brasil. \\ ${ }^{\text {b}}$ Programa de Pós-graduação em Ciências Fisiológicas, Universidade Federal do Pampa, \\ Uruguaiana, RS, Brasil. \\ ${ }^{\mathrm{c} C}$ Curso de Medicina, Universidade Federal do Pampa, Uruguaiana, RS, Brasil.
}

Histórico do Artigo Recebido em: 03/11/2017

Aceito em:

$30 / 03 / 2018$

\section{Palavras-Chave:}

Sistema Único de Saúde; terapias complementares; capacitação profissional.

Key words: Unified Health System; complementary therapies; professional training.

\begin{abstract}
RESUMO
A Política Nacional de Práticas Integrativas e Complementares (PNPIC) no Sistema Único de Saúde (SUS) contempla sistemas médicos complexos e recursos terapêuticos, com ênfase na escuta acolhedora, no desenvolvimento do vínculo terapêutico e na integração do ser humano com o meio ambiente e a sociedade. O presente relato de experiência tem como objetivo compartilhar as estratégias utilizadas pelo Ambulatório de Práticas Integrativas e Complementares no SUS, para a implementação das Práticas Integrativas e Complementares (PICs), bem como da capacitação dos recursos humanos produzidos pela universidade. Essa experiência foi gerada a partir do projeto de extensão "Ambulatório de Práticas Integrativas e Complementares no SUS". O projeto constitui-se de capacitações realizadas por docentes e especialistas, e atendimentos aos usuários oriundos do SUS que apresentavam dor musculoesquelética, pelos acadêmicos participantes das capacitações. Foram realizadas aproximadamente 15 capacitações no ano de 2017 sobre diversos temas envolvendo PICs. Nelas, participaram 116 sujeitos e 94,67\% deles aprovaram as qualificações. Ainda foram realizados mais de 250 atendimentos e os pacientes relataram redução significativa $(p<0,001)$ do seu nível de dor após os atendimentos com PICs com média da Escala Visual Analógica (EVA) 6,81 antes e EVA 1,23 depois da intervenção. Concluiu-se que as capacitações contribuíram muito para a formação dos participantes, bem como os atendimentos apresentaram bons resultados de melhora da dor dos pacientes, o que contribui para que continuemos com ambas as ações, visando à ampliação delas.
\end{abstract}

Proposal for a teaching-service intervention of Integrative and Complementary Practices

\section{ABSTRACT}

The National Policy on Integrative and Complementary Practices (PNPIC) in the Unified Health System (SUS) contemplates complex medical systems and therapeutic resources, with an emphasis on welcoming listening, developing the therapeutic link and integrating the human being with the environment and society. The present experience report aims to share the strategies used by the Ambulatory of Integrative and Complementary Practices in the SUS, for the implementation of Integrative and Complementary Practices (PICs), as well as the training of the human resources produced by the University. This experience was generated from the extension project "Ambulatory of Integrative and Complementary Practices in SUS". The project consists of trainings - carried out by teachers and specialists, and treatment to users from the SUS that presented musculoskeletal pain, by the academics participating in the training. Approximately 15 training sessions were held in 2017 on various to PICs involving PICs. 116 subjects participated and $94.67 \%$ of them approved the qualifications. More than 250 patients were treated and reported a significant reduction $(\mathrm{p}<0.001)$ in their pain level after treatment with the mean of (Analogic Visual Scale) EVA 6,81 before and EVA 1,23 after the intervention. We concluded the training contributed a lot to the participants, as well as, the attendances presented good results of improvement of the patients' pain, which contributes to that we continue with both actions, aiming at the expansion of them.

\footnotetext{
*Autor correspondente: angelazanella@ unipampa.edu.br (A. K. Zanella)
} 


\section{Introdução}

No final da década de 70, a Organização Mundial de Saúde (OMS) criou o Programa de Medicina Tradicional, com objetivo de formular políticas nessa área. A partir disso, a OMS expressou, em vários comunicados e resoluções, o seu compromisso em incentivar os Estados-membros a formularem e implementarem políticas públicas para uso racional e integrado da medicina tradicional e complementar/alternativa (MT/MCA) nos sistemas nacionais de atenção à saúde, bem como para o desenvolvimento de estudos científicos para melhor conhecimento de sua segurança, eficácia e qualidade (1).

No Brasil, a legitimação e a institucionalização da MT/MCA foram iniciadas a partir da década de 80, principalmente, após a criação do Sistema Único de Saúde (SUS). Com a descentralização e a participação popular, os estados e municípios ganharam maior autonomia na definição de suas políticas e ações em saúde (2). Além disso, desde 1985, convênios entre Instituições e Universidades, resoluções, decretos presidenciais, entre outros eventos e documentos, auxiliaram na regulamentação e construção dessa política pública (3). Ademais, o Ministério da Saúde apresentou, em 2006, a Política Nacional de Práticas Integrativas e Complementares (PNPICS) no SUS, cuja implementação envolve justificativas de natureza política, técnica, econômica, social e cultural. Essa política atende à necessidade de se conhecer, apoiar, incorporar e implementar experiências, destacando-se a Medicina Tradicional Chinesa-Acupuntura, Homeopatia, Fitoterapia, Medicina Antroposófica e Termalismo-Crenoterapia (3).

A PNPICS contempla sistemas médicos complexos e recursos terapêuticos, envolvendo abordagens que buscam estimular os mecanismos naturais de prevenção de agravos e recuperação da saúde por meio de tecnologias eficazes e seguras, com ênfase na escuta acolhedora, no desenvolvimento do vínculo terapêutico e na integração do ser humano com o meio ambiente e a sociedade. Além disso, existe uma visão ampliada do processo saúde-doença e a promoção global do cuidado humano, especialmente do autocuidado $(1,3,4)$. Para institucionalização das práticas complementares, a PNPICS desencadeou o desenvolvimento de políticas, programas, ações e projetos, levando ao SUS o que antes era restrito aos usuários da assistência complementar de saúde (2).

Atualmente, os profissionais de Práticas Integrativas e Complementares (PICs) e generalistas consideram a abordagem holística nas concepções de saúde e cuidado relativas às PICs como forma de diminuir efeitos colaterais das intervenções da medicina convencional e aumentar a satisfação do paciente. Afirma-se que a efetivação da PNPIC pode favorecer a consideração da singularidade dos usuários e famílias, além de aumentar a satisfação com a qualidade da atenção recebida. Isso porque os profissionais de saúde que mantêm contato com as PICs acabam ampliando suas concepções de saúde e cuidado. Essa ampliação pode contribuir para o reconhecimento respeitoso das diferentes racionalidades explicativas da saúde e cuidado, na perspectiva da interculturalidade na atenção à saúde e, nessa medida, contribuir para uma abordagem mais integral do processo saúde-doença (2).

A PNPIC trouxe avanços para a saúde no Brasil pela normatização e institucionalização das experiências com essas práticas na rede pública, fato comprovado pelo aumento significativo de investimentos em consultas e procedimentos das PICs (de 4672945,75 reais em 2007 para 8032782,46 reais em 2011), da oferta de produtos e serviços (134 estabelecimentos públicos com PICs cadastrados) e da aprovação de políticas, programas e legislação nas três instâncias de governo durante a formulação e após a aprovação dessa política (1).

As Práticas Integrativas e Complementares (PICs) têm por objetivo a prática 
profissional mais humanizada e a visão integral do paciente, realizando ações de promoção e prevenção em saúde e de tratamento de doenças. Uma das vantagens do manejo com as PICs é que são meios terapêuticos mais simples e baratos e que possuem eficácia comprovada tornando a implementação das PICs na Atenção Primária à Saúde (APS) fundamental (5).

Nesse contexto - apesar de ainda ser limitado o acesso a esses recursos no Brasil vem crescendo a quantidade de instituições (de ensino, pesquisa e extensão) interessadas no tema $(6,7)$. Normalmente, oferta-se os serviços como auriculoterapia, acupuntura, fitoterapia, homeopatia, tratamentos antroposóficos, uso medicinal de fontes termais, de plantas e de águas minerais com características medicinais, dependendo da disponibilidade de recursos humanos, naturais e materiais (2). Partilhando de tal interesse, o Projeto de Extensão "Ambulatório de Práticas Integrativas e Complementares no SUS" oferece capacitações e atendimentos gratuitos à comunidade externa e acadêmica da Universidade Federal do Pampa (UNIPAMPA).

A UNIPAMPA é uma instituição multicampi no estado do Rio Grande do Sul (RS) fundada em 2008. Teve por objetivo de implantação desenvolver a região da fronteira oeste, que, anteriormente, era classificada como área de repulsão populacional devido a sua intensa estagnação econômica. O campus da saúde encontra-se na cidade de Uruguaiana que, devido a sua posição estratégica (cidade fronteiriça), o desenvolvimento no setor de saúde e a sua economia são maiores que as cidades vizinhas. Entretanto, o município apresenta um total aproximado de 130 mil habitantes, os quais, em sua maioria, apresentam baixo poder aquisitivo e baixa escolaridade (4), fatores que contribuem para dificultar o acesso à saúde e à educação.

Diante dessa realidade, este relato visa compartilhar as estratégias utilizadas pelo Ambulatório de Práticas Integrativas e Complementares no SUS, para a implementação das PICSs em um município da fronteira oeste do Rio Grande do Sul, bem como da capacitação dos recursos humanos produzidos pela universidade, a fim de promover um olhar mais ampliado do processo do cuidado e atenção à saúde, voltado para a proposta da PNPICS, que valoriza a humanização e integralidade no cuidado dos profissionais de saúde aos usuários do SUS.

\section{Materiais e Métodos}

O presente estudo possui delineamento longitudinal, descritivo e analítico, sendo que o público-alvo do estudo foi composto por adultos com idade entre 20 a 55 anos. A proposta do projeto foi desenvolvida em duas etapas: a primeira, composta por capacitações aos acadêmicos e comunidade; a segunda etapa foi composta pelos atendimentos com PICs, em que a amostra era composta por homens e mulheres com lesões ocupacionais, identificados pelas Unidades Básicas de Saúde da cidade de Uruguaiana. Estima-se que a população nessa faixa etária na cidade é de 63.219 adultos (4), sendo que estes foram classificados de acordo com sua queixa principal e graduação da dor.

Trata-se de um artigo do tipo relato de experiência, originado a partir de demandas de indivíduos com doenças crônicas na cidade de Uruguaiana, que não conseguiam atendimentos nos postos de saúde. Essa experiência foi gerada a partir do projeto de extensão "Ambulatório de Práticas Integrativas e Complementares no SUS", proveniente da Universidade Federal do Pampa (UNIPAMPA). O ambulatório localizado dentro da instituição possui salas e ambiente climatizados, equipados com materiais para a realização de diversas PICs, entre elas: acupuntura, auriculoterapia, massoterapia, reiki, moxaterapia, ventosaterapia, entre outras. As atividades desse 
programa foram iniciadas em março de 2016 e permanece ativo até o presente momento - início de 2018. No entanto, os dados do presente trabalho são referentes aos dados do primeiro e segundo semestre de 2017.

O projeto constituiu-se em duas etapas, que ocorreram concomitantemente: capacitações e atendimentos (Figura 1). Ambas as ações ocorreram mais de uma vez, totalmente gratuitas e os ministrantes convidados se propuseram a participar como voluntários.

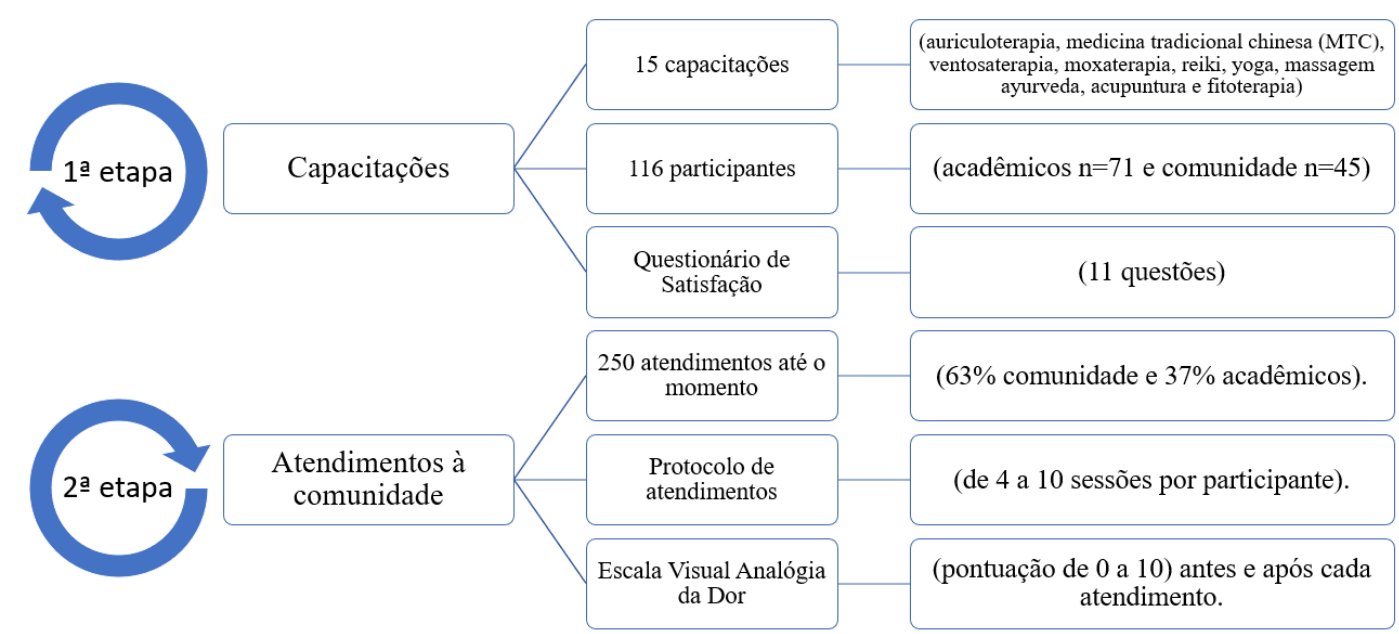

Figura 1: Organograma de atividades do projeto de extensão Ambulatório de Práticas Integrativas e Complementares no SUS.

As capacitações foram realizadas por docentes dos cursos de medicina, medicina veterinária e fisioterapia da UNIPAMPA, especialistas nos temas abordados e por profissionais experts, indicados pela comunidade e oriundos do Brasil e da Argentina, nos laboratórios e dependências da própria Instituição de ensino. Entre os temas abordados nas capacitações, destacamos: auriculoterapia, medicina tradicional chinesa (MTC), ventosaterapia, moxaterapia, reiki, yoga, massagem ayurveda, acupuntura e fitoterapia, sendo importante ressaltar que algumas capacitações não permitem a realização de atividades práticas pelos alunos, como o reiki, ou então não habilitaram os alunos para realizarem qualquer atendimento (MTC, reiki e yoga), pois esses procedimentos exigem uma formação mais específica e o principal objetivo da atividade foi promover o momento de vivência, de debate e divulgação dessas técnicas e dos conhecimentos.

Ao final de cada capacitação, os participantes foram convidados a preencher um Questionário de Satisfação. Esse questionário apresentava onze perguntas com o objetivo de avaliar a conduta do ministrante (pontualidade, didática, interesse e conduta ética, por exemplo), verificar a aplicabilidade prática dos conhecimentos obtidos após cada capacitação, bem como a nota representativa da satisfação dos participantes sobre as capacitações. Além disso, no questionário, estavam presentes questões para averiguar o quanto os participantes estavam interessados em continuar aprendendo sobre o tema e se eles se sentiam seguros para aplicar o conhecimento adquirido.

$\mathrm{Na}$ segunda etapa do projeto, foram realizados atendimentos $(\mathrm{n}=250)$ aos usuários oriundos do SUS, selecionados a partir de demanda espontânea. Os atendimentos foram divulgados por meio de cartazes nas Estratégias de Saúde da Família (ESFs) do município e por meio digital, através das redes sociais. Os critérios de inclusão utilizados foram possuir lesões por esforços repetitivos (LER) e/ou distúrbios osteomusculares relacionados ao trabalho (DORT). Para cada participante, foi delineado 
um protocolo de atendimento específico, de 4 a 10 atendimentos, considerando suas queixas e principais sintomas a serem tratados, o que deixa claro a visão individualizada que as PICs possibilitam a cada paciente (4).

As técnicas utilizadas durante os atendimentos, realizadas pelos acadêmicos, com supervisão da coordenadora, foram: auriculoterapia; ventosaterapia; liberação miofascial; massagem relaxante; pedras quentes; meditação induzida; aromaterapia; e cromoterapia. Antes e após os atendimentos, os pacientes eram questionados sobre a intensidade da dor/queixa que sentiam, tendo como parâmetro, a Escala Visual Analógica (EVA). A EVA foi explicada pelos acadêmicos ao paciente, o qual relatou sua dor de acordo com uma escala ilustrada e numerada de 0 (nenhuma dor) até 10 (máximo de dor) (8).

\section{Resultados e Discussão}

Foram realizadas 15 capacitações no ano de 2017, porém esse cronograma é contínuo e as temáticas são definidas de acordo com as sugestões dos participantes e dos especialistas atuantes no projeto. As atividades são abertas para participação da comunidade externa e acadêmica da UNIPAMPA e dos municípios da tríplice fronteira. As capacitações contribuíram para: (1) ampliar o conhecimento dos participantes sobre as PICs; (2) oferecer práticas das atividades ministradas (sempre que possível); (3) divulgar as PICs para a comunidade; (4) promover momentos de educação permanente aos profissionais da saúde; e (5) estimular os acadêmicos a realizar novos estudos acerca dos temas trabalhados.

Participaram das capacitações 116 sujeitos, sendo eles alunos dos cursos da área da saúde da UNIPAMPA (n=71) (medicina, farmácia, fisioterapia, educação física, medicina veterinária e enfermagem) e pessoas interessadas no assunto oriundas da região $(n=45)$. Esses participantes receberam conteúdos teóricos e vivências práticas sobre avaliação, análise, observação e propostas de intervenções em doenças crônicas e em suas variações. A grande procura de acadêmicos na busca de obter conhecimento sobre as PICs nos remete ao fato de que, há alguns anos, parecia haver falta de profissionais habilitados nessa área, bem como a baixa oferta de serviço especializado ou pouco conhecimento e interesse dos próprios profissionais de saúde para orientar os pacientes (9).

Estudos apontam que os profissionais de saúde até apresentam interesse sobre PICs, pois a maior parte acredita que essas práticas podem contribuir na melhoria da saúde. Porém, sua experiência se torna limitada devido à carência de capacitação teóricoprática nessa área e por ignorarem a legislação vigente (10). Esse tema nos leva à hipótese de que, na rede pública, especialmente na atenção primária, a maior parte dos profissionais não empregam as PICs por não possuírem capacitação e/ou formação para atender a crescente demanda por essas práticas (11), o que tornou a atividade realizada no projeto atual ainda mais relevante. Ademais, os acadêmicos da UNIPAMPA demonstraram grande interesse nessa área, reconhecendo essa prática como uma atividade que pode ser desenvolvida para o atendimento e bem-estar do seu paciente.

Muitos dos participantes relataram que seu conhecimento sobre PICs era escasso antes das capacitações. Sabe-se que o desconhecimento sobre as PICs, seus benefícios e também seus riscos pode levar o profissional da saúde a criar certa resistência em indicar essas terapias à população (12). Porém, observando os dados colhidos a partir dos questionários de satisfação após as qualificações foi possível verificar 77,09\% de notas máximas nas capacitações. Ou seja, os participantes demonstraram uma grande satisfação com o aprendizado sobre as PICs, mesmo sem apresentarem uma base mais 
consolidada sobre o tema. Espera-se que, em um futuro próximo, os acadêmicos do projeto tornem-se profissionais com um pensamento mais amplo sobre a relação saúdedoença e sobre os tratamentos que poderão ser utilizados nos seus pacientes.

Com relação à aplicabilidade das atividades na rotina profissional (Gráfico 1), os resultados também foram satisfatórios, com $94,67 \%$ de aprovação em todas as capacitações. Algumas técnicas aprendidas nas capacitações são relativamente fáceis de serem aplicadas, levando ao reconhecimento, difusão e a aplicação das PICs e possibilitando um tratamento de fácil acesso e baixo custo ao paciente. Estudos indicam que grande parte das PICs, como a homeopatia, fitoterapia, acupuntura, chás medicinais, massoterapia, yoga e shantala, são aceitos e utilizados nas Unidades Básicas, sendo sua aplicação influenciada pelo baixo custo, pelo fato de se acreditar que efeitos colaterais são inexistentes, satisfação e crença da população $(13,14,15)$.

No entanto, ainda esbarramos em diversos desafios de sua aplicabilidade, especialmente no que tange à necessidade de formação específica para atuar com essas técnicas (8). Esse é o caso do reiki, que apresentou $89,36 \%$ de aplicabilidade relativamente menor que os demais. Estudos reforçam a necessidade da ampliação da capacitação das PICs tanto no serviço de saúde como também nas instituições de ensino $(16,17)$, a fim de promover a saúde e qualidade de vida dos usuários do SUS, a partir de profissionais qualificados para tal (19).

Gráfico 1: Avaliação da aplicabilidade prática dos conhecimentos alcançados a partir das capacitações de acordo com o questionário de satisfação respondido pelos participantes (n=116). Os dados obtidos nessa análise são referentes ao ano de 2017.

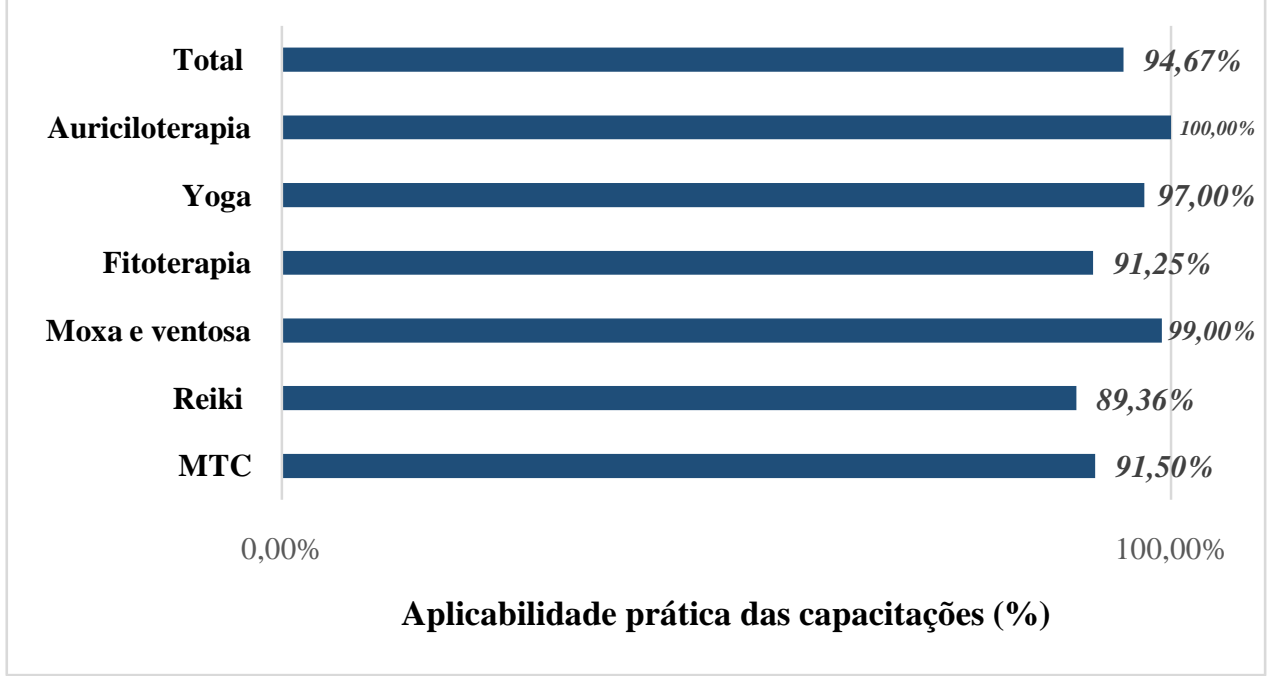

Na Tabela 1, estão descritas as notas de satisfação dos participantes nas capacitações, sendo possível perceber que todas as capacitações tiveram a pontuação 5, considerada nota máxima de satisfação. No apanhado geral, é possível observar que 85,34\% dos participantes, em todas as capacitações, classificou a satisfação com nota 5, 8,63\% com nota 4 e $4,31 \%$ com nota 3 . Esses dados, reforçam a importância da realização das capacitações aos profissionais da saúde, sendo uma possível forma de valorização da integralidade no cuidado em saúde. As PICs possuem um potencial importante na valorização do cuidado integral em saúde e das discussões da Saúde Coletiva, estimulando a transição do modelo hegemônico biomédico para a promoção da saúde (18). No entanto, ainda observamos o despreparo técnico dos profissionais da saúde atuantes na assistência, bem como faltam ações de formação que preparem os mesmos (19). 
Tabela 1: Frequência da nota representativa da satisfação dos participantes das capacitações das PICSs, obtido a partir do questionário de satisfação após a realização de cada capacitação (Ano 2017).

\begin{tabular}{ccccccc}
\hline Capacitação & 5 & 4 & 3 & 2 & 1 \\
\hline MTC1 & 13 & 1 & 0 & 1 & 1 \\
MTC2 & 17 & 2 & 1 & 0 & 0 \\
Reiki 1 & 13 & 1 & 1 & 0 & 0 \\
Moxaterapia e & 15 & 1 & 0 & 0 & 0 \\
ventosaterapia & 14 & 0 & 1 & 0 & 0 \\
Reiki 2 & 6 & 2 & 1 & 0 & 0 \\
Fitoterapia & 12 & 1 & 1 & 0 & 0 \\
Yoga & 9 & 2 & 0 & 0 & 1 \\
Auriculoterapia & 99 & 10 & 5 & 1 & 0,86 \\
\hline Total & & & & & \\
\hline
\end{tabular}

Até o presente momento, foram realizados mais de 250 atendimentos no projeto de extensão, sendo $63 \%$ para a comunidade externa da instituição e $37 \%$ aos acadêmicos) ambos oriundos de demanda espontânea do SUS, executados pelos discentes participantes das capacitações sob supervisão e atuação dos profissionais especialistas nas técnicas supracitadas. Os indivíduos que participaram como pacientes neste projeto de extensão apresentavam dores osteomusculares em diferentes locais do corpo, mas todos foram avaliados segundo a EVA. Sabemos que a dor é definida pela Associação Internacional para o Estudo da Dor como uma "experiência sensorial e emocional de caráter desagradável provocada por lesão tissular, ou atribuída a tal". Dessa forma, a dor possui uma sinalização que envolve estruturas periféricas e/ou centrais do sistema nervoso, que é um componente fisiológico da dor. Porém, a dor possui um componente emocional que é extremamente importante e que contribui para a ampliação ou redução na percepção desse sintoma (20).

Alguns autores relatam que estas contribuem para a redução da ansiedade, proporcionando relaxamento e contato com seus sentimentos e emoções $(21,22)$. Com o uso das PICs, pode-se levar os dois aspectos da dor em consideração e tratar esse problema de forma eficaz. Esse fato foi evidenciado pela observação de que, com as PICs, os indivíduos com dor musculoesquelética tiveram uma redução da dor, quando as avaliações da EVA são comparadas antes e após aos 250 atendimentos bastante significativos $(p<0,05$, Tabela 2$)$. Esse resultado corrobora com estudos que apontam que o tratamento com terapias complementares proporciona ao usuário melhora da dor e da medicalização, tornando-se uma ótima estratégia para a redução do uso descontrolado de analgésicos e antidepressivos (18, 23, 24,25).

Tabela 2: Média da Escala Visual Analógica (EVA) antes e após os atendimentos ( $\mathrm{n}=250)$ com PICSs no ano 2017.

\begin{tabular}{c|c|c|c}
\hline EVA antes & EVA depois & D.P & $\mathrm{p}^{*}$ \\
\hline 6,81 & 1,23 & 2,34 & $<0,05$ \\
\hline
\end{tabular}

*Teste t de student para amostras pareadas.

A partir da experiência vivenciada, foi possível verificar o sucesso dessas atividades com relação aos objetivos a elas foram propostos, afinal elas foram frequentadas por alunos dos cursos de graduação de medicina, farmácia, enfermagem e fisioterapia, alunos de pós-graduação, residentes do Programa de Residência Multiprofissional em Saúde e comunidade externa. 
As medicinas alternativas podem estar ocupando o lugar deixado vago pela medicina convencional e dispõem de muita experiência a transmitir nesse sentido (23). A compartimentação da medicina atual em áreas cada vez mais especializadas acentua ainda mais o distanciamento entre o profissional da saúde, especialmente o médico, e o indivíduo, levando a um pensamento simplório da doença, como se sua importância fosse menor do que de fato é. Dessa forma, as PICs possuem um objetivo divergente dos objetivos da assistência alopática e têm contribuído para a qualidade de vida de seus usuários, na medida em que visam à assistência e à saúde do indivíduo, considerando-o como mente/corpo/espírito e não um ser fragmentado (26).

Levando-se em conta que, segundo a OMS, saúde é um estado de completo bem-estar físico, mental e social, e não somente a ausência de enfermidade ou invalidez, um tratamento mais amplo, considerando o indivíduo como um todo parece mais adequado. No entanto, é importante salientar que não se pretende sobrepor as PICs em relação à medicina tradicional nem substituir nenhuma outra prática existente. Isso porque as diversas formas terapêuticas possuem seu valor e aplicação. Sendo que as PICs vêm para somar junto a elas.

\section{Considerações finais}

A partir das experiências e dos resultados colhidos, foi possível observar que as capacitações contribuíram muito para a formação dos participantes, bem como os atendimentos apresentaram bons resultados de melhora da dor dos pacientes, o que contribui para que continuemos com ambas as ações, visando à ampliação delas. Essa ampliação é pretendida por meio da maior divulgação dos encontros formativos, da criação de materiais de apoio dos temas abordados nas capacitações, da extensão dos convites para mais profissionais, docentes e comunidade externa participarem no ambulatório. Buscar-seá, também, a criação de uma disciplina optativa que tenha como tema central as PICs na universidade e o estabelecimento de uma parceria com a Secretaria Municipal de Saúde que permita a implementação das PICs nas ESFs da cidade.

Conflito de interesses: Os autores declaram não haver conflito de interesses.

\section{Referências}

1. Brasil. Ministério da Saúde. Secretaria de Atenção à Saúde. Departamento de Atenção Básica. Práticas integrativas e complementares: plantas medicinais e fitoterapia na Atenção Básica/Ministério da Saúde. Secretaria de Atenção à Saúde. Departamento de Atenção Básica. - Brasília: Ministério da Saúde, 2012.

2. World Health Organization. Acupuncture: Review and analysis of reports on controlled clinical trials. Geneva: WHO Publications, 2002.

3. Brasil. Ministério da Saúde. Secretaria de Atenção à Saúde. Departamento de Atenção Básica. Política Nacional de Práticas Integrativas e Complementares no SUS - PNPICS-SUS/Ministério da Saúde, Secretaria de Atenção à Saúde, Departamento de Atenção Básica. - Brasília: Ministério da Saúde, 2006.

4. Instituto Brasileiro de Geografia (IBGE). Estimativas da população residente com data de referência. D.O.U em 28/08/2014. Rio de Janeiro: IBGE-DEPIS. Disponível em:www.ibge.gov.br. Acesso em 02 de novembro de 2017.

5. Schveitzer MC, Zoboli ELCP. Papel das práticas complementares na compreensão dos profissionais da Atenção Básica: uma revisão sistemática. Rev Esc Enferm USP 2014; 48(Esp):189-196.

6. Trippo KV, Almeida L, de Jesus MC, Nascimento MS, Moreira NL, Oliveira M C. Concepção de Acadêmicos de Saúde sobre a PNPIC e Sua Aplicabilidade no SUS. Revista Pesquisa em Fisioterapia 2017; 7(4): 481-488. 
7. Fischborn A, Machado J, Fagundes N, Pereira N. A Política das Práticas Integrativas e Complementares do SUS: o relato da implementação em uma unidade de ensino e serviço de saúde. Cinergis, 2016; 17: 17(4 Supl.1):358-363.

8. Santos MC, Tesser CD. Um método para a implantação e promoção de acesso às Práticas Integrativas e Complementares na Atenção Primária à Saúde. Ciênc. Saúde Coletiva 2012;17(11):3011-3024.

9. Chaitow L. Guia do terapeuta: massagem para dor lombar e pélvica. Rio de Janeiro: Elsevier, 2008.

10. Fontanella F, Speck FP, Piovezan AP, Kulkamp IC. Conhecimento, acesso e aceitação das práticas integrativas e complementares em saúde por uma comunidade usuária do Sistema Único de Saúde na cidade de Tubarão/SC. Arq Cat Med 2007;36(2):69-74.

11. Manzini T, Martinez EZ, Carvalho ACD de. Conhecimento, crença e uso de medicina alternativa e complementar por fonoaudiólogas. Rev Bras Epidemiol 2008;11(2):304-314.

12. Sousa IMC, Bodstein RCA, Tesser CD, Santos FAZ, Hortale VA. Práticas integrativas e complementares: oferta e produção de atendimentos no SUS e em municípios selecionados. Cad Saúde Pública 2012;28(11):2143-2154.

13. Neves RG, Pinho LB, Gonzáles RIC. O Conhecimento dos Profissionais de Saúde Acerca do Uso de Terapias Complementares no Contexto da Atenção Básica. Rev Pesqui Cuid Fund 2012;4(3):25022509.

14. Nagai SC, Queiroz MS. Medicina complementar e alternativa na rede básica de serviços de saúde: uma aproximação qualitativa. Ciênc. Saúde Coletiva 2011;16(3):1793-1800.

15. Loures MC, Porto CC, Siqueira KM, Barbosa MA, Medeiros M, Brasil VV, Pereira MAD. Contribuições da fitoterapia para a qualidade de vida: percepções de seus usuários. Rev enferm 2010;18(2):278-283.

16. Randow R, Campos KFC, Roquete FF, Hanashiro LT, Duarte VES, de Almeida Guerra V. Periferização das práticas integrativas e complementares na atenção primária à saúde: desafios da implantação do Lian Gong como prática de promoção à saúde. Revista Brasileira em Promoção da Saúde 2017; 29: 111-117.

17. Losso LN, Freitas SFTD. Avaliação do grau da implantação das práticas integrativas e complementares na Atenção Básica em Santa Catarina, Brasil. Saúde em Debate 2017; 41: 171-187.

18. Paranaguá TTB, Bezerra ALQ, Souza MA, Siqueira KM. As práticas integrativas na estratégia saúde da família: visão dos agentes comunitários de saúde. Rev Enferm 2009;17(1):75-80.

19. Azevedo E, Pelicioni MCF. Práticas integrativas e complementares de desafios para a educação. Trab educ saúde 2011; 361-378.

20. De Simoni C, Benevides I, Barros NF. As práticas integrativas e complementares no SUS: realidade e desafios após dois anos de publicação da PNPICS. In: BRASIL. Ministério da Saúde. Revista Brasileira Saúde da Família. Práticas integrativas e complementares em saúde: uma realidade no SUS. 2008.

21. Loeser JD, Treede RD. The Kyoto protocol of IASP Basic Pain Terminology. Pain 2008;137:473-477.

22. Papa MAB, Dallegrave D, Pereira AG. Práticas integrativas e complementares em centros de atenção psicossocial como ampliação do cuidado em saúde. Rev Sau Red 2017;2(4):409-417.

23. Franco SC, Ferreira BA, Patrício BMS, da Costa RMO, Gomes FEV, Coutinho BD. A acupuntura e as práticas integrativas e complementares no SUS sob a ótica da fisioterapia. Encontros Universitários da UFC 2017;1(1):4168.

24. Souza LP, Teixeira FL, Diniz AP, de Souza AG, Delgado LHV, Vaz AM, Vieira PMO, Rodriguez PS. Práticas Integrativas e Complementares no cuidado à saúde mental e aos usuários de drogas. Id on Line Revista Multidisciplinar e de Psicologia 2017; 11(38): 177-198.

25. Kurebayashi LFS, Turrini RNT, de Souza TPB, Takiguchi RS, Kuba G, Nagumo MT. Massagem e Reiki para redução de estresse e ansiedade: Ensaio Clínico Randomizado. Revista Latino-Americana de Enfermagem, 2016: 24: 1-8.

26. Luz MT. Cultura Contemporânea e Medicinas Alternativas: novos paradigmas em saúde no fim do século XX. PHYSIS: Saúde Col. 2005;15(Sup):145-176. 